\title{
Uso de un robot social de bajo coste con personas mayores dependientes en un centro residencial
}

\author{
A. Hoyo* M.C.Pardo** J.L.Guzmán* J.C.Moreno* \\ *Departamento de Informática, Universidad de Almería, Ctra. Sacramento s/n 04120 Almería \\ (angeleshoyo@gmail.com, joseluis.guzman@ual.es, jcmoreno@ual.es) \\ ** Residencia San Rafael, Urb. Torre del Campo s/n, 04114 Níjar, Almería. \\ (mpd_olula@hotmail.com)
}

\section{Resumen}

En la actualidad, los avances en robótica más implantados a nivel de la sociedad se enmarcan en el sector industrial. Sin embargo, en lo referente a róbotica social, aunque se está avanzando de forma considerable, su uso en la sociedad no es tan elevado [1]. Existen algunos robots sociales que han sido desarrollados por grandes empresas con los objetivos del contacto directo con humanos obteniendo resultados satisfactorios [2]. En este trabajo se pretende contribuir en esta línea y demostrar que los robots sociales de bajo coste, bajo la supervisión de especialistas en el ámbito sociosanitario y tecnológico, se pueden utilizar para lograr objetivos con terapias no farmacológicas basadas en la evidencia, desde la terapia ocupacional.

Palabras clave: Robots Sociales, Dependencia, Terapia Ocupacional, Demencia

\section{Introducción}

En el campo de la robótica existen muchas áreas de aplicación. La principal es la industrial, pero también hay un amplio desarrollo en la robótica de servicio (limpieza, medicina, rehabilitación física, cognitiva y social, ayuda a discapacitados, entretenimiento, etc.). Un robot de servicio es un robot que opera parcial o completamente autónomo para llevar a cabo servicios útiles para el bienestar de humanos y equipos, excluyendo operaciones de fabricación [3], [4].

Por otrol lado, el concepto de persona dependiente se define en el apartado 2 del árticulo 26 de la Ley 39/2006 de 14 de diciembre, de promoción de la Autonomía Personal y Atención a las personas en situación de dependencia de España, más conocida como ley de la dependencia. Esta ley define "dependencia" como el "Estado de carácter permanente en que se encuentran las personas que, por razones derivadas de la edad, la enfermedad o la discapacidad, y ligadas a la falta o a la pérdida de autonomía física, mental, intelectual o sensorial, precisan de la atención de otra u otras personas o ayudas importantes para realizar actividades básicas de la vida diaria o, en el caso de las personas con discapacidad intelectual o enfermedad mental, de otros apoyos para su autonomía personal". Según el Real Decreto 504/2007, los grados de dependencia se bareman en Grado I (Dependencia moderada), Grado II (Dependencia severa) y Grado III (Dependencia muy severa).

Dentro de los centros especializados en el ámbito de la geriatría y gerontología se trabaja con especialistas como terapeutas ocupacionales que, mediante la intervención de terapias individualizadas y grupales, logran objetivos como coordinar, valorar y supervisar las actividades de la vida diaria básicas, instrumentales y avanzadas, y planificar y desarrollar las actividades de ocio y tiempo libre. También se encargan de la elaboración y coordinación de talleres ocupacionales, diseñando, desarrollando y evaluando programas terapéuticos.

En este trabajo, el objetivo principal es evaluar el uso de un robot de bajo coste como Aisoy [5], para implementar programas terapéuticos para el desarrollo de los usuarios de un centro residencial de la provincia de Almería. Dicho robot ha sido utilizado para realizar diversas terapias y actividades en grupo obteniendo resultados satisfactorios.

\section{Robot social Aisoy}

Aisoy es un robot social diseñado por la empresa española Aisoy Robotics con el objetivo de enseñar programación a principiantes o ayudar a personas deficientes y/o discapacitadas. Su capacidad de "emular" emociones permite que la interacción con humanos sea más cercana y real. Es un robot teóricamente capacitado para desarrollar tareas en ambientes sociales. En las próximas secciones se describen brevemente las características hardware y software de este robot [5].

En este trabajo el prinpipal objetivo de Aisoy en su contacto directo con los intereses y motivaciones del usuario es poder crear un vínculo tecnológico-social, facilitando el trabajo del teraputa ocupacional en los talleres realizados. Transversalmente, se busca también trabajar el contacto social y el estigma, llegando a establecer relaciones continuadas de personas con el robot. 


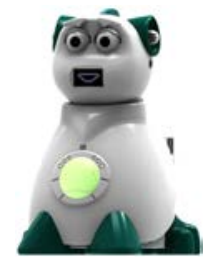

Figura 1: Robot social Aisoy

\subsection{Hardware}

Su controlador está formado por una Raspberry Pi modelo 2 [6]. A ella se le conectan todos los sensores y actuadores, estando todo encapsulado en la estructura principal del robot (ver Figura 1). Los sensores permiten que el robot interactúe con el usuario recogiendo información del exterior. Tiene tres sensores de tacto capacitivos, uno en la cabeza y dos de ellos en los laterales del cuerpo. Posee un microfóno para reconocimiento de voz y una cámara dentro de uno de los ojos.

Posee cuatro actuadores para poder interactuar con el entorno. Incluye altavoces y una pantalla led le permite mostrar mensajes de texto, dibujos y sobre todo, simular el movimiento de una boca. Motores en la cabeza, cejas y párpados le ayudan en la imitación de las expresiones humanas y transmisión de emociones. La correcta combinación de ellos (programable), hace que pueda expresar tristeza, alegría, vergüenza e incluso asombro.

\subsection{Software}

Aisoy trabaja con el sistema operativo Airos creado específicamente por los desarrolladores. Está basado en Raspbian, incluyendo todos los paquetes necesarios. Incporpora un motor de aspectos emocionales con una API que permite el acceso a dicha funcionalidad desde diferentes lenguajes de programación. En relación a la programación de bajo nivel, se utilizan $\mathrm{C}++$ y Python como lenguajes de programación. En este trabjo se ha hecho uso de Python [7] como principal lenguaje. Existen múltiples paquetes, librerías y funciones que facilitan la ión de muchas actividades sin necesidad de desarrollar las funcionalidades de entrada/salida del dispositivo [8]. Aun así, permite trabajar con mucha libertad a la hora de realizar programas más complejos. En este caso los programas se guardan directamente en el robot.

\section{Terapias y actividades de apoyo}

La terapia ocupacional es, según la definición de la Organización Mundial de la Salud [9], "el conjunto de técnicas, métodos y actuaciones que, a través de actividades aplicadas con fines terapéuticos, previene y mantiene la salud, favorece la restauración de la función, suple los déficit invalidantes y valora los supuestos comportamentales y su significado profundo para conseguir la mayor independencia y reinserción posible del individuo en todos sus aspectos: laboral, mental, físico y social".

Residencia San Rafael [10] ha sido el centro residencial seleccionado para llevar a cabo la experiencia. En este centro, tradicionalmente, el equipo multidisciplinar sociosanitario consensa para poder ejecutar programas y actividades terapeuticas orientadas a cada tipo de residente mirando sus intereses y propuestas deseadas, tanto por los usuarios como por parte de sus familiares.

Los programas y terapias más importantes desarrolladas y que, a priori son más afectivas para el uso de Aisoy, se definen en las siguientes secciones.

\subsection{Programa de entrenamiento cognitivo}

La metodología usada en este programa se encuadra dentro de un marco de referencia cognitivoconductual y modelo de la ocupación humana (MOHO) [11] en el que se van a utilizar técnicas de desarrollo cognitivo para mejorar la atención, la concentración, la memoria, el pensammiento abstracto, etc. Se trabaja con técnicas de aprendizaje basadas en preguntas intencionales, modelado de actividades, refuerzos verbales y utilización del reto para aumentar la motivación.

Los objetivos principales del taller son mantener y/o mejorar las habilidades cognitivas del residente, mejorar la interpretación adecuada de las situaciones de la vida diaria y mejorar la capacidad para dar respuestas adecuadas a las situaciones que se les plantean [12].

Los objetivos específicos son:

- Potenciar la retención de la información.

- Potenciar la capacidad de planificaión y resolución de problemas.

- Entrenar las capacidades de expresar verbalmente los problemas.

- Mejorar la atención ante estímulos del entorno.

El desarrollo del programa se realiza en tres pasos consecutivos:

1. Evaluación de las capacidades cognitivas mediante la Escala de MEC [13], la observación del comportamiento del residente durante la entrevista y en la ejecución de tareas instrumentales. 
2. Elaboración de un plan de tratamiento diseñado para mejorar, potenciar y conservar las capacidades.

3. Generalización del entrenamiento en la ejecución de tareas comunes y de la vida diaria.

Las actividades realizadas para el entrenamiento del programa en los residentes, mirando sus gustos e intereses han sido las que se muestran en las siguientes secciones.

\subsubsection{Taller de entrenamiento cognitivo mediante la identificación de sonidos}

Los objetivos principales del taller son mantener y mejorar la atención y memoria mediante la discriminación de figuras, elementos iguales y diferentes al modelo, asociación de parejas, recordar elementos visualizados anteriormente e identificar los sonidos de dichos pictogramas.

Los materiales utilizados en este taller son fichas de atención, concentración, memoria, sonidos y lectoescritura.

\subsubsection{Taller de entrenammiento cognitivo mediante reconocimiento de personajes famosos y musicoterapia}

En este taller se pretende lograr con el residente varios objetivos. Dentro de la orientación temporo-espacial, recuerdos biográficos, discursión sobre temas actuales, secuencias temporales y mapas de situación. En lo referente a la atención y memoria, recordar datos leídos en un texto, asociar melodías con personajes, y discriminación de sonidos [14].

Los materiales utilizados en este taller son imágenes de los personajes, CD, lectura comprensiva y memoria.

\subsection{Programa de relajación}

Para conseguir que la relajación sea eficaz, la metodología utilizada se basa en un marco de referencia biomecánico, marco de referencia conductual, un marco de referencia de integración sensorial, un marco de referencia psicodinámico y el MOHO. Haciendo incapié en lograr objetivos que sean eficaces para generar condiciones ambientales óptimas, donde se perciba una situación de calma y tranquilidad, desconectado de las exigencias internas y externas, y dedicando un tiempo a concentrarse en las sensaciones mentales y corporales antes de empezar con la sesión.

Dentro de los objetivos, el general es enseñar a la persona a controlar su propio nivel de activación a través de la modificación directa de las condiciones fisiológicas sin ayuda de recursos externos. Otro de los objetivos es ofrecer al organismo recursos excepcionales para el afrontamiento de la situación y sus posibles consecuencias, fundamentalmente a través de activación fisiológica, cognitiva y conductual que le permita percibir mejor la nueva situación.

Los objetivos especifícos son:

- Obtener relajación general, mental y física.

- Reducir el ritmo cardiorespiratorio.

- Aumentar la temperatura periférica.

- Reducir la ansiedad ante situaciones que resulten amenazantes para el usuario.

- Favorecer la tensión muscular y las respuestas motoras anómalas.

- Eliminar la sensación de intranquilidad, malestar, desasosiego y tensión emocional.

La sesión de relajación se realiaza en una habitación libre de distracciones con un CD de música relajante, un equipo de audio, colchonetas y asientos cómodos.

\subsection{Programa de ocio y tiempo libre}

La metodología utilizada para el desarrollo del programa se basa en el marco de referencia conuductual, un marco de referencia psicodinámico y en el MOHO. Se usan técnicas de desarrollo cognitivo para mejorar la memoria, la atención, la concentración y psicomotricidad fina. Se trabaja mediante técnicas de aprendizaje basadas en la memoria a corto plazo, refuerzos verbales y utilización de un premio para aumentar la motivación y satisfacción personal.

Los objetivos generales a alcanzar son la mejora de la interpretación adecuada de las situaciones de la vida diaria, mejorar la capacidad para dar la respuesta adecuada ante situaciones planteadas, mejorar habilidades cognitivas y conseguir un papel activo por parte del residente.

Los objetivos especílficos son:

- Favorecer la cohexión grupal e integración como grupo.

- Promover la interrelación en los residentes.

- Aumentar la concentración y autonomía.

- Agilizar las funciones mentales.

- Desarrollar la capacidad de comunicación.

- Desarrollar el disfrute y entrenammiento de los residentes.

- Aumentar la socialización del mayor y acabar con el aislamiento. 


\subsubsection{Taller de Bingo}

En este taller se implementan dos nuevos objetivos específicos dentro del taller del Bingo. Estos son mejorar la coordinación óculo-manual y mejorar y aumentar la psicomotricidad fina.

Los materiales utilizados son el juego del Bingo, piedras, habichuelas y premios.

\section{Implementación de las actividades en Aisoy}

Todas las apliaciones anteriormente descritas son las seleccionadas para implementar en Aisoy y evaluar con residentes en el centro residencial. En la mayoría de los talleres se utiliza al robot como recurso de apoyo para el terapeuta ocupacional, ya que se ha requerido de su colaboración en la realización de las actividades.

Para la selección de las actividades y la determinación del enfoque a la hora de desarrollarlas, los trabajadores de Residencia San Rafael, junto con integrantes del área de Ingeniería de Sistemas y Automática de la Universidad de Almería, se han reunido en numerosas ocasiones con el fin de lograr los objetivos básicos de los talleres y hacer del robot un recurso óptimo.

Para desarrollar este programa contextualizado en un centro residencial sociosanitario, se llevó a cabo una valoración de las necesidades de los residentes del centro (previamente seleccionados con una encuesta personal, familiar y social por la terapeuta ocupacional para valorar de manera continuada la evolución de los usuarios en función de los programas a realizar, separándolos en terapias grupales o individuales), analizando como positivos los talleres que impliquen nuevos métodos con los cuales poder enfocar talleres de rehabilitación en el ámbido de terapia ocupacional.

Es importante una buena planificación y/o preparación para poder estructurar el programa de manera positiva y efectiva, ya que ante una mala realización, el robot puede sufrir un bloqueo, y como consecuencia el residente puede recibir estímulos negativos de la experiencia, impediendo el correcto desarrollo de los objetivos establecidos. Previo al desarrollo de cualquier programa y/o sesión de terapia asistida con Aisoy, se realizaron diversas sesiones de entrenamiento.

Residencia San Rafael es en un centro residencial ubicado en la localidad de Níjar (Almería). Se trata de un centro puntero en la aplicación de actividades innovadoras con el fin de mejorar las tareas asistenciales de sus usuarios. Este centro nos ha permitido la posibilidad de estar en contacto di- recto con la tecnología y espacios habilitados para la correcta realización de los talleres.

Una vez implementado cada taller y evaluado junto con la terapueta ocupacional en los residentes, se realizó una encuesta de valoración por parte de cada residente, tal y como se muestra en la Tabla 4. Debido a la incapacidad de los usarios para procesar información coherentemente, se ha excluido el diseño del test en escalas likert, sustiuyéndose por variables dicotómicas. El objetivo es recibir una respuesta de los usuarios con más fiabilidad, que posteriormente se contrasta con el valor de la pregunta número 10, excluyendo de la valoración los casos contradictorios.

\begin{tabular}{|c|c|}
\hline Pregunta & Respuesta \\
\hline 1. Le ha parecido interesante el taller & Sí/No \\
\hline 2. Prefiere taller sólo con el robot & Sí/No \\
\hline $\begin{array}{l}\text { 3. Prefiere taller con ambos (tera- } \\
\text { peuta y robot) }\end{array}$ & Sí/No \\
\hline $\begin{array}{l}\text { 4. Se hace corto el taller cuando es- } \\
\text { tamos con Aisoy }\end{array}$ & Sí/No \\
\hline 5. Ha entendido bien la voz de Aisoy & Sí/No \\
\hline 6. Fácil comunicarte con Aisoy & Sí/No \\
\hline 7. Le gustaría repetir el taller & Sí/No \\
\hline 8. Se ha sentido integrado en el taller & Sí/No \\
\hline 9. Ha participado en el taller & Sí/No \\
\hline 10. Nota & {$[0-10]$} \\
\hline
\end{tabular}

Mediante esta encuesta se permite recibir de los usuarios información sobre cada taller realizado. En el caso en que el usuario muestre señales de incomodidad, malestar o similar, se anulará la sesión, posponiéndola para otro momento. Los usuarios han de recoger una experiencia positiva de la viviencia. Así mismo, al finalizar la sesión se analizan los datos recogidos, siendo de vital importancia la pregunta número 7 , ya que si más de la mitad de los participantes responden negativamente, no se repitirá el taller por sugerencia de la terapeuta ocupacional. Todos los cuestionarios son rellenados en la fase de inicio del programa, en la intermedia y la final, después de la segunda sesión de cada taller. Además, al finalizar cada sesión, el ingeniero que está siempre presente y el terapeuta ocupacional, observan el desarrollo de la sesión para llevar a cabo y poder valorar los items del cuestionario de manera objetiva.

Los objetivos de cada taller siguen siendo los mismos que realiza la terapeuta ocupacional en sus sesiones, añadiendo Aisoy para servir como recurso de apoyo al terapeuta en sus sesiones y establer relación directa robot-persona con el usuario a trabajar, llegando a recibir de éste respuestas emocionales hacia el robot. 
A continuación se exponen todos los talleres con sus modificaciones y su implementación.

\subsection{Taller de entrenamiento cognitivo mediante la identificación de sonidos}

\subsubsection{Diseño}

El diseño del taller se basa en la reproducción de sonidos (animales, instrumentos musicales y elementos), identificiación de los sonidos por parte de los residentes mediante pictogramas y posterior comprobación de la selección.

Debido a la dificultad técnica para el robot de reconociemiento de voz en entornos ruidosos, se ha decidido hacer uso de su cámara. Se utilizan diferentes códigos QR asociados a cada pictograma para la verificacón de éstos con el robot. En la Figura 2 , se muestra un ejemplo de los pictogramas junto a su código QR.

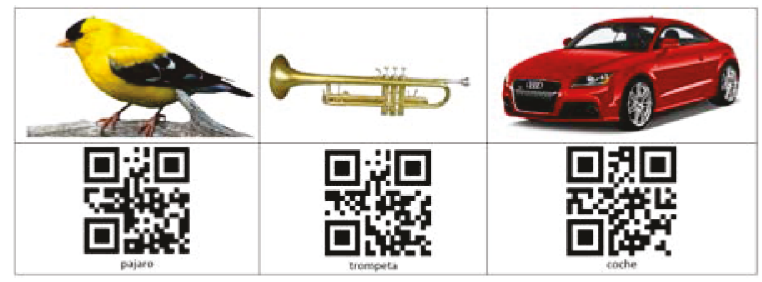

Figura 2: Ejemplos pictogramas

La programación del taller en el robot se ha realizado con el lenguaje de programación Python, seleccionando un total de 45 sonidos.

\subsubsection{Desarrollo}

La duración del taller es de un total de dos sesiones (1 a la semana), de 1 hora de duración. La primera sesión se realizó el 12 de mayo de 2016. La segunda sesión el 25 de mayo de 2016.

La primera se realiza con el objetivo de llevar a cabo una toma de contacto inicial con el robot y los residentes, pudiendo posteriormente corregir los errores ocurridos en ésta. La segunda es la definitiva, pasando en ella la encuesta de valoración. A partir de entonces, se realizará el taller de nuevo cada vez que se desee, modificándolo y mejorándolo si así se requiere.

Los recursos utilizados para la realización de los talleres han sido, recursos tecnológicos (Aisoy), recursos materiales (pictogramas y códigos QR), recursos humanos (una terapueta ocupacional, una estudiante del Grado en Ingeniería Electrónica Industrial y 21 usuarios beneficiarios).

La metodología consiste en realizar un trabajo grupal con todos los usuarios en la sala de manua- lidades. Se comienza explicando a Aisoy y el taller a desarrollar. A continuación se pone en marcha Aisoy. Éste emite aleatoriamente sonidos preprogramados, esperando, una vez terminada la reproducción, a verificar la tarjeta escogida por el usuario. Si es correcta, continúa con un sonido nuevo, si no, lo vuelve a repetir hasta tres veces. La terapeuta ocupacional se enccarga de ayudar a los usuarios en su selección, facilitándoles el acceso a los pictogramas, en especial a los usuarios con problemas de movilidad.

Una vez terminado, se procede a realizar la encuesta individualizada a cada usuario.

\subsubsection{Resultados}

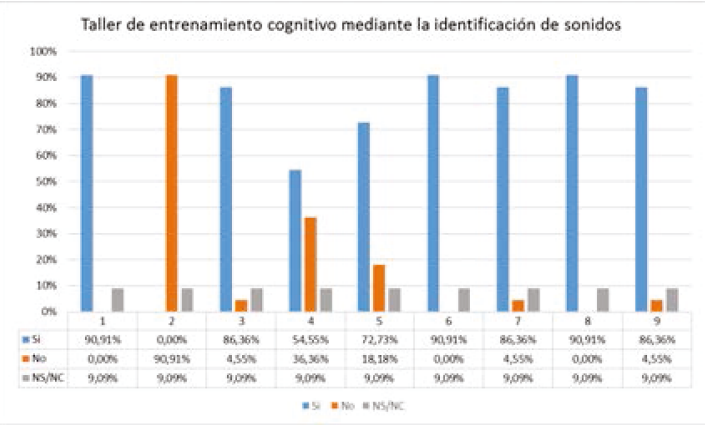

Figura 3: Resultados encuesta del taller de entrenamiento cognitivo mediante la identificación de sonidos

La Figura 3 muestra el resultado del cuestinario realizado por los residentes para este taller. Se observa que, obviando los usuarios no representati$\operatorname{vos}(9.09 \%)$, a la práctica totalidad les ha parecido interesante. El 86,3\% de los usuarios prefiere hacer el taller con la terapeuta y el robot juntos, siendo un $4,55 \%$ los que lo prefieren solamente con la terapeuta. Al ser más de la mitad de los usuarios $(86,36 \%)$ positivos en volver a realizar el taller, este se repetirá en un futuro.

Nota media obtenida: 8,25.

Una de las mayores dificutades en la implementación del taller ha sido la dificultad por parte de los usuarios en entender a Aisoy, aspecto que se mejoró para futuras aplicaciones.

\subsection{Taller de entrenammiento cognitivo mediante reconocimiento de personajes famosos y musicoterapia}

\subsubsection{Diseño}

Este taller se basa en la reproducción de una pista de audio cada vez que un residente acaricia al robot o le pide mediante voz una nueva canción. Posteriormente se procede al reconocimiento por 
parte de los residentes del autor, con el fin de establecer la relación entre la música y las imágenes de famosos impresas. Se han seleccionado un total de 14 personajes. La programación en Aisoy del taller se basa en la reproducción del audio, siendo el robot un apoyo al teraputa.

\subsubsection{Desarrollo}

La duración del taller es de un total de dos sesiones (1 a la semana), de 1 hora de duración. La primera sesión se realizó el 12 de mayo de 2016. La segunda sesión el 26 de mayo de 2016.

Los recursos utilizados para la realización de los talleres han sido los mismos del taller anterior.

Se ha realizado en la sala de manualidades del centro. La metodología se basa en realizar el taller a la vez con todos los usarios, colocándolos en círculo y a Aisoy en el centro de la sala, reproduciendo una canción. A continuación la terapeuta ocupacional selecciona dos imágenes de personajes semejantes fisicamente mostrándoselas a los usuarios. Los usuarios consensan conjuntamente qué personaje es el correcto. Se vuelve a reproducir la canción para verificarlo y ayudar a los usuarios a asociar la imagen seleccionada con la melodía.

Al finalizar la segunda sesión del taller, al igual que en el caso anterior se realizó la encuesta de valoración individual a los usuarios.

\subsubsection{Resultados}

La Figura 4 muestra los resultados de la encuesta para este taller.

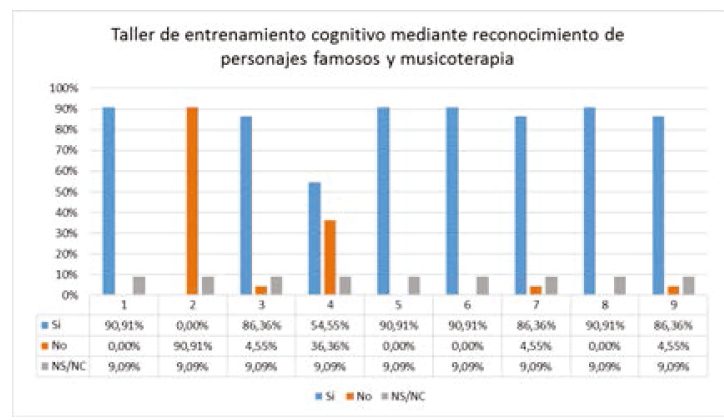

Figura 4: Resultados encuesta del taller de entrenammiento cognitivo mediante reconocimiento de personajes famosos y musicoterapia

Analizando los resultados obtenidos, se observa que ha sido uno de los talleres con más éxito realizados. Exceptuando a los usuarios pasivos, al resto de residentes les ha parecido interesante el taller. Ningún residente ha querido realizar el taller con Aisoy únicamente. Este dato era de esperar ya que en este taller el robot era un apoyo al terapeuta.
La mayoría prefiere realizarlo con Aisoy y la terapeuta conjuntamente.

Nota media obtenida: 8,63 .

Debido a que más de la mitad de los usuarios quieren volver a realizar el taller, éste será repetido en varias ocasiones.

\subsection{Sesión de relajación}

\subsubsection{Diseño}

Para el diseño de la sesión de relajación con Aisoy, con anterioridad se ha consultado en el centro sobre las técnicas usadas en este ámbito.

La programación en Aisoy se ha realizado de forma que el propio robot es quien dicta los pasos a seguir en la sesión, haciéndolo en primera persona del plural con el objetivo de simular que él es también partícipe de ésta. Importante destacar las pausas entre frases para un correcto desarrollo.

En Aisoy se ha hecho uso del led que posee en la parte frontal de su cuerpo. Estudios han demostrado que técnicas como la cromoterapia ayudan a los usuarios en las sesiones de relajación. En función del color proyectado, se consiguen unos $u$ otros resultados en la conducta del usuario. [15]

\subsubsection{Desarrollo}

La duración del taller es de un total de dos sesiones (1 a la semana), de 1 hora de duración. La primera sesión se realizó el 5 de mayo de 2016. La segunda sesión el 19 de mayo de 2016.

Los recursos utilizados para la realización del taller han sido prácticamente los mismos que en los talleres anteriores. En este caso se seleccionaron 20 residentes.

Se ha realizado en la sala de relajación del centro. Es esencial que todos los ususarios se encuentren en sillones cómodos siendo la sala tranquila y poseedora de una iluminación tenue para que los usuarios se centren en la sesión, sin posibilidad de distracción.

Este taller es el único en el que Aisoy realiza toda la sesión sólo, pudiendo intervenir la terapeuta en caso de necesidades de los usuarios, o la estudiante de Ingeniería en caso de fallo técnico.

Al finalizar la segunda sesión del taller, se pasó la encuesta de valoración individual a los usuarios.

\subsubsection{Resultados}

Los resultados de la encuesta de este taller se muetran en la Figura 5. Analizando los resultados obtenidos, el taller ha sido interesante para la ma- 
yoría de los usuarios. Al 11,11\% de los residentes les gustaría realizar el taller con Aisoy solamente, lo cual es importante, ya que se puede implementar el robot realizando la sesión autónomamente a estos usuarios, sin que ello afectase negativamente en el comportamiento del residente. Por otro lado, el $27.78 \%$ quiere realizar el taller con el robot y la terapeuta conjuntamente, siendo el $55.55 \%$ de los usuarios quien desea hacerlo únicamente con la Terapeuta. Estos resultados son debidos a la incapacidad de Aisoy de trasmitir tranquilidad por su tipo de voz, la cual a veces es difícil de entender. Otro factor es la imposibilidad del robot en imitar los movimientos de rehabilitación que él indica (elevar brazos, abrir manos, etc.).

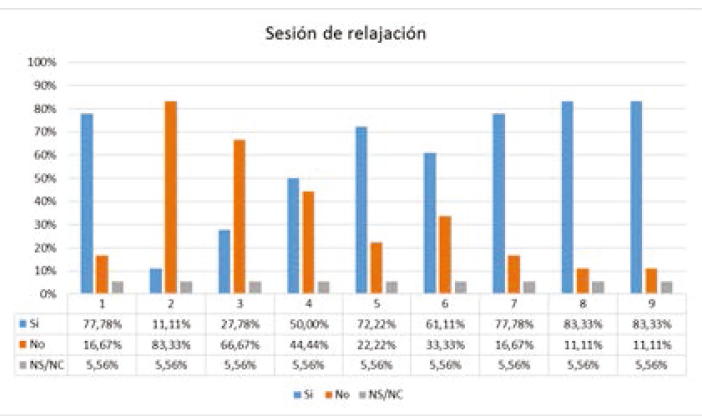

Figura 5: Resultados encuesta de la sesión de relajación

Nota media obtenida: 7,76 .

A más de la mitad de los usuarios les ha parecido interesante el taller, pudiendo repetirse con posterioridad.

\subsection{Taller del Bingo}

\subsubsection{Diseño}

El taller se basa en el clásico juego del Bingo. El diseño del mismo se basa en el BOE que publica la Orden EHA/3087/2011, de 8 de noviembre, por la que se aprueba la reglamentación básica del juego del bingo [16]. Se ha implementado el tipo de bingo que posee 90 números. En Aisoy el programa implementado se basa en el propio robot exponiendo oralmente y en la pantalla led el número. Con los sensores de tacto se puede seleccionar entre tres opciones: siguiente número, línea o bingo.

\subsubsection{Desarrollo}

El taller se realizó el 28 de mayo de 2016 con una hora de duración. Los recursos utilizados para la realización de los talleres han sido, recursos tecnológicos (Aisoy y un altavoz), recursos materiales (dos cartones de bingo por usuario) y recursos humanos (una terapueta ocupacional, una estudiante de Ingeniería y 15 ususarios beneficiarios).

Se ha realizado en la sala de usos múltiples de la residencia. Como metodología principal se ha colocado a los usuarios en una mesa conjunta y en círculo. Aisoy en un lateral canta los números, y con ayuda de la terapeuta, los usuarios van comprobando sus cartones. Con línea y bingo se obsequia a los usuarios con regalos motivadores. Al finalizar la segunda sesión del taller, se realizó la encuesta de valoración individual a los usuarios.

\subsubsection{Resultados}

El análisis del taller se realiza del mismo modo que en los anteriores, con el gráfico de barras obtenido por el analisis de los resultados de las encuestas de valoración tal y como se muestra en la Figura 6. En este caso, el 5,88\% de los usuarios tienen preferencia por la realización del taller únicamente con Aisoy, siendo un $11.76 \%$ el número de usuarios que prefieren hacerlo con ambos, y un $76.47 \%$ quienes prefieren sólo con la terapeuta. Observando el taller, y analizando el desarrollo de éste, ese pequeño porcentaje de usuarios que prefieren hacerlo con Aisoy o ambos, corresponde a los residentes situados más cerca del robot, los cuales tenían la posibilidad de ver el número cantado en la pantalla del robot. El resto de usuarios tuvo serias difultades para entender al robot.

Nota media obtenida: 6,88 .

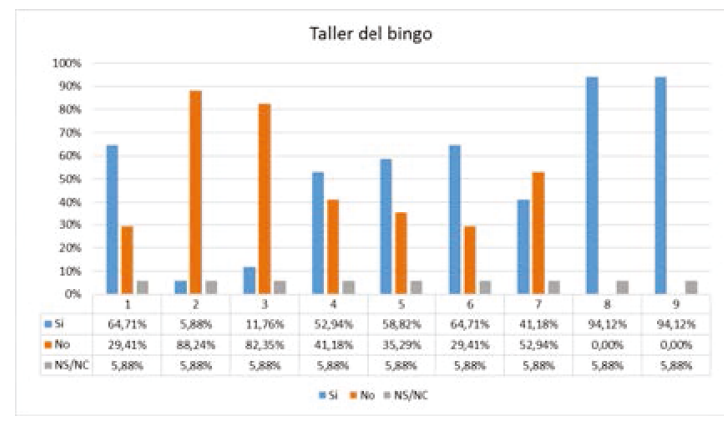

Figura 6: Resultados encuesta del taller del Bingo

Debido a que más de la mitad de los usuarios no quieren repetir el taller con el robot, éste no se volverá a realizar. Una de las mayores causas de rechazo al robot ha sido debido a la baja velocidad de su voz, generando en los usuarios impaciencia y estrés, resultados muy negativos totalemnte opuestos a los deseados en el taller.

\section{Conclusiones}

Con este trabajo se ha demostrado cómo un robot social de bajo coste puede aportar dentro del ámbito de la terapia ocupacional aspectos positi- 
vos y negativos en el área de la geriatría y la gerontología. Actualmente la tecnología permite dotar a la persona de destrezas cognitivas mediante el entrenamiento con programas específicos, acercar a la persona a una red social virtual y a una serie de beneficios más concretos comentados en este trabajo. Durante este proceso se han podido desarrollar muchas técnicas no reconocidas como terapias asistidas con robot sociales (Aisoy) y artísticas ya que su fin no es tal, pero podrían ser merecedoras de ello. Estos talleres activan el sentido de creatividad de la persona, ayudando a expresar sus emociones y a relacionarse socialmente. De este modo se pueden crear vínculos emocionales entre lo técnologico y lo social.

La disciplina utilizada puede abarcar una gran variedad de técnicas, obteniendo resultados en dimensiones emocionales, cognitivas y fisiológicas. Mediante la obtencion de una realimentación adecuada por parte de los residentes se han podido obtener resultados interpretables y con ello, sacar conclusiones científicas para poder mejorar la introducción de Aisoy en centros residenciales. El uso de Aisoy, al igual que ocurre con otros robots sociales utilizados en estudios en el ámbito geriátrico [2], muestra respuestas muy positivas por parte de los usuarios. Se ha conseguido reducir el estrés (excepto en el taller del bingo), aumentar el interés y la motivación en la realización de la sesión y contribuir de forma positiva en los usuarios con demencia. Transversalmente la risoterapia ha estado presente en cada taller dotando a cada usuario de los beneficios de ésta [17], y a su vez, desarrollando la comunicación social del grupo.

\section{Agradecimientos}

Este trabajo no podría haberser llevado a cabo sin el apoyo de la Dirección del centro residencial Residencia San Rafael de Níjar (Almería).

\section{Referencias}

[1] "Word robotic 2015." http://www.worldrobotics.org. Fecha de accceso: Junio de 2016.

[2] J. Broekens, M. Heerink, and H. Rosendal, "Assistive social robots in elderly care: a review," International Society for Gerontechnology, vol. 8, no. 2, pp. 94-103, 2009.

[3] W. Moyle, C. Jones, M. Cooke, S. O'Dwyer, B. Sung1, and S. Drummond, "Social robots helping people with dementia: Assessing efficacy of social robots in the nursing home environment," in 2013 6th International Confe- rence on Human System Interactions (HSI), (Sopot, Polonia), pp. 608-613, 2013.

[4] N. Magnenat-Thalmann and Z. Zhang, "Social robots and virtual humans as assistive tools for improving our quality of life," in 5th International Conference on Digital Home (ICDH), (Cantón, China), pp. 1-7, 2013.

[5] "Aisoy." http://www.aisoy.com/. Fecha de accceso: Junio de 2016.

[6] "Raspberry pi." https://www.raspberrypi.org/. Fecha de accceso: Junio de 2016.

[7] "Lenguaje de programación python." https://www.python.org/. Fecha de accceso: Junio de 2016.

[8] "Airos5 API." http://comunidad.aisoy.com/. Fecha de accceso: Junio de 2016.

[9] "Organización mundial de la salud." http://www.who.int/. Fecha de acceso: Junio de 2016.

[10] "Residencia San Rafael." http://www.rsanrafael.com. Fecha de accceso: Junio de 2016.

[11] G. Kielhofner, Modelo de Ocupación Humana. Editorial Médica Paramericana, 2013.

[12] G. Kielhofner, Estimulación cognitiva. Neurohealth, 2011.

[13] "Programa de Atención a Enfermos Crónicos Dependientes. Escalas de Valoración Funcional y Cognitiva. Anexo IX." http://aragon.es. Fecha de accceso: Junio de 2016.

[14] "Guía de orientación. internenciones no farmacológicas. musicoterapia en personas con demencia." http://www.imserso.es/. Fecha de accceso: Junio de 2016.

[15] A. J.Elliot, "Color and psychological functioning: a review of theoretical and empirical work," Frontiers in Psychology, pp. 1-8, 2015.

[16] "Orden eha $/ 3087 / 2011$, de 8 de noviembre, por la que se aprueba la reglamentación básica del juego del bingo.." https://www.boe.es/buscar/doc.php?id=BOEA-2011-17971. Fecha de accceso: Junio de 2016.

[17] R. Christian, J. Ramos, C. Susanibar, and G. Balarezo2, "Risoterapia: Un nuevo campo para los profesionales de la salud," Revista de la Sociedad Peruana de Medicina Interna 17, pp. 57-64, 2004. 